Vol.:2 Issue:2 Date:December 2018 Received: 17.11.2018 Accepted: 07.12.2018 Final Version: 25.12.2018

ISVOS Journal 2018, 2(2):136-147

\title{
Complementary coded waveforms sets in Marine Radar Application
}

\author{
V. Koshevyy ${ }^{a, 1}$, V. Tryhuba ${ }^{b}$ \\ ${ }^{a, b}$ National University “Odessa Maritime Academy”, Odessa, Ukraine
}

\begin{abstract}
Complementary coded waveform and mismatched filter pairs sets are used. On the contrary with Golays matched waveform filter pair the mismatched waveform filter pair does exist for all $\mathrm{N}$ (number pulses in waveform). Using corresponding shapes of filters and proper chosen of waveforms Doppler tolerance may be provided. This property together with a good range side-lobs level suppression makes it's attractable for use in marine radar.
\end{abstract}

Keywords: "Composite signal, Ambiguity function, Cross-ambiguity function, Amplitude modulation, Periodic mode"

\section{Introduction}

Application of pulse compression waveforms gives the possibility increasing the range of radar operation under limited transmitter peak power; improving detectability small-size targets on the background sea surface by mean increasing Doppler selectivity. Extraction of signals from interfering reflections is very important for such kind of radar waveform and filter design. The quality of such extraction significantly depends from range-velocity distribution of interfering reflections [1, 2, 3]. The problem of the mismatched filter and waveform design that maximizes the signal-to-noise-plus-clutter ratio at the receiver filter output has been formulated and addressed in [4], [5], [6], [7], [8], [9], [10], [11]. Mismatched filtering may causes degradations in signal-to-white noise ratio. In [10] the method of a filter optimization which maximizes the signal-to-noise ratio under additional quadratic constraints was developed. In [12] the methods of joint optimization signal and filter for interfering reflections suppression under additional constraints on range resolving performance, signal-to-noise ratio loses and given amplitude modulation of signal with different limitations on the memory and the width of the pass band of the filter were developed. A key problem in phase coded with a unimodular sequence is the presence of range sidelobes in the ambiguity function of the coded waveforms. Range sidelobes from a strong interfering reflector can result in masking of nearby weak targets. It is however impossible to design a single unimodular sequence for which the aperiodic cross correlation function has no range sidelobes by the methods presented in issues we were pointed above. This has led to the idea of using complementary sets of unimodular sequences $[13,14]$ for phase coding. Complementary sequences have the property that the sum of their autocorrelation functions vanishes at all delays other than zero.

The effective ambiguity function of Golay pair of phase coded waveforms, for example, is free of range side lobes along the zero-Doppler axis. However, this ideal property is sensitive to Doppler effect. The ambiguity function of Golay pairs for phase coded waveforms off the zero-Doppler axis has large range side lobes [2].

The ambiguity function of a pulse train of complementary waveforms, in which two or more waveforms are transmitted alternatively in time over several pulse repetition intervals (PRIs), suffers from the same problem. The sensitivity of complementary waveforms to Doppler has been a major barrier for using these waveforms for radar pulse compression.

Thus the construction a Doppler resilient pulse train of complementary waveforms is very important task for modern radars. In [15] was proposed a new multi-channel radar scheme employing polarization diversity for obtaining multiple independent views of the target. In this scheme, Golay pairs of phase coded waveforms are used to provide synchronization while Alamouti coding [16] is used to coordinate transmission of these waveforms on the horizontal and vertical polarizations. The combination of Goley complementary sequences and Alamouti coding makes it possible to do unambiguous radar polarimetry on a pulse-by-

\footnotetext{
${ }^{1}$ Corresponding author. Tel.: +0-000-000-0000 ; fax: +0-000-000-0000 .

E-mail address: vmkoshevyy@gmail.com
} 
pulse basis. But this method requires special polarization processing technique. In [17] a Doppler compensation scheme that exploits the structure of the received waveform matrix constructed on the base of the Golay complementary pair of sequences.

It was shown that the received waveform matrix can be processed in a way to minimize effects of Doppler.The drawbacks of this approach is the some complexity of the process of minimization the effects of Doppler. The attractive approach is reported in [18] where was considered coordinating the transmission of Golay complementary pairs coded waveforms in time by ProuhetThue-Morse (PTM) sequence (over time repetition intervals) to construct Doppler resilient pulse trains, for which the ambiguity function is effectively free of range side lobes inside a desired Doppler interval.

The signals and filters design technique presented in [19] is extension of methods [12] to the case of waveforms and filters sets with group-complementary properties, which are optimized simultaneously. From other hand in [26] was shown that if for given signal some level of suppression of cross ambiguity function (CAF) in given range-Doppler zone is provided for Doppler shifting this zone the same signal is provided the same level of CAF suppression only by means appropriate chosen of filter. It is true also for complementary coded waveforms and for any Doppler shift value. More over it is true as for filters, which optimized for one cross section, so for the filters optimized in Doppler strip. For unknown Doppler shift may be used multichannel Doppler filter. Although multichannel construction partially resolves the problem of unknown between periods Doppler shift case compensation, in the case of two or more targets with closed different Doppler shifts possibility of their mutual harmful influence are still remained. That's why the needs of suppression range side lobes in Doppler strip are still actual. For this purpose methods of optimization [19,20] for set of filters (or set of waveforms and filters) may be used. Because these methods not only allows to provide group complementary properties, but also provides a possibility for clutter suppression with given range-Doppler distribution (including Doppler strip distribution) as well in contradistinction to the methods considered in $[24,25]$. In this paper, we describe more simple approach for construction a Doppler compensation scheme that is constructed in three steps. On the first step a set of waveforms and corresponding filters with complementary property is calculated. On the second step multichannel Doppler scheme for compensation over period Doppler shift phases is chosen according to [26]. At the third stage it is suggested that a few blocks of waveforms and filters with group-complementary properties in each block, which were chosen at the first step are used. Each block contains the same waveforms and corresponding filters, but the order of their disposition in each block may be different and is chosen for provided decreasing range side-lobes level in Doppler strip. In this work we consider discrete signals and the optimizing discrete filters for cases electronically scanning antenna and mechanical scanning rotating antenna. The approach for Doppler shift compensation is pointed above may be used for both cases [27].

We consider discrete signals and the optimizing discrete filters with complex envelopes [19, 20]:

$$
\begin{aligned}
& S(t)=\sum_{p=1}^{P} \sum_{n=0}^{N-1} S_{p n} u\left(t-(p-1) T-n T_{0}\right) \\
& W(t)=\sum_{p=1}^{P} \sum_{m=0}^{M-1}\left(W_{p m} u\left(t-(p-1) T-m q_{f} T_{0}\right)\right.
\end{aligned}
$$

where $u(t-m Z)=\left\{\begin{array}{l}1,(\mathrm{~m}-1) \mathrm{Z} \leq t \leq m c Z \\ 0, \text { for other values of } \mathrm{t}\end{array} ;\right.$

$T_{0}$ - is elementary pulse $\mathrm{u}(\mathrm{t})$ duration; $\mathrm{T}-$ repetition period of signals; $q_{f}=\Delta F_{S} / \Delta F_{W}-$ is a parameter which characterizes the pass-band of the filter $\Delta F_{W}$ in respect to the spectral width of the signal $\Delta F_{S}$;

$S_{p n} ; W_{p m}$ - are complex amplitudes and weighting coefficients of waveform $S_{p}$ and mismatched filter $W_{p}$ pairs.

$P$ - number of waveform and mismatched filter pairs in set.

Considering optimization reduces to a choice of the signal $S(t)$ and of the filter $W(t)$ which maximizes the ratio [12]:

$$
\sigma=\frac{A^{2} \mid X_{S W}(0,0)^{2}}{v \int_{-\infty}^{+\infty}|W(t)|^{2} d t+\sigma_{0} \iint_{-\infty}^{+\infty} \sigma_{\xi}(\tau, f)\left|X_{S W}(\tau, f) I\right|^{2} d \tau d f}
$$

Where;

$$
X_{S W}(\tau, f)=\int_{-\infty}^{+\infty} W^{*}(t) S(t-\tau) e^{i 2 \pi f t} d t
$$


is the Cross ambiguity function of signal (1) and filter $(2) ; \sigma_{\xi}(\tau, f)=\sigma(\tau, f)+\xi \delta(f) ; \sigma(\tau, f)$ is the range - velocity distribution of the interfering reflections; $\delta(f)$ is delta-function; $A, \sigma_{0}$ are parameters which characterizes the reflecting properties of the target and the interfering reflections; $\nu, \xi$ are the parameters for controlling signal-to - noise ratio loses and range resolving properties correspondingly. Moreover, for the considered below problem of multiple joint waveforms and filters optimization, by a proper parameter selection, the ideal correlation properties (no range side-lobes) for the sum of cross-correlation functions (complementary property) may be enforced.

\section{Filter and Waveform Optimization}

We consider the case when,

$$
N T_{0}+M q_{f} T_{0} \leq T \text { (if } q_{f}=1, M=N, \text { so } 2 N T_{0} \leq T \text { ) }
$$

In the case (5) expression (4) can be written as follows (see [19], [20],[21],[22])

$$
X_{S W}(\tau, f)=\sum_{p=1}^{p} X_{S_{p} W_{p}}(\tau, f) e^{i 2 \pi f(p-1) T}
$$

Formula (6) simplifies the task of maximizing (3). So we have got expression (3) for the sets of signals and filters at the same form as we have for the single signal and filter and we can use iteration process of joint optimization signal and filter which was described in [12]. The iteration process is follows: at first for the given sets of signals we are getting optimal sets of filters, then for this sets of filters we are getting optimal sets of signals and so on. The convergence of the iteration process was proved in [23].

Ratio (3) for signal (1) and filter (2) with using (6) may be rewrite in the matrix form

$$
\sigma=\frac{A^{2}|W * S|^{2}}{W^{*}\left[v I+\sigma_{0} D_{{ }_{\xi}}\right] W}
$$

Where $S, W$ are the vectors of complex amplitude of signals set and filters set; $I$ - identity matrix; $D_{\theta \xi}$ is correlation matrix of interfering reflections with range-velocity distribution $\sigma_{\xi}(\tau, f)$.

At the first iteration of optimization process we choose any initial set of signals vector $S^{0}$ and find for it optimum vector sets of filters $W^{0}$ according to expression [12]

At the next iteration for the $W^{(0)}$ we find $S^{(1)}$ [12]

$$
W^{(0)}=\left[v I+\sigma_{0} D_{\theta \xi}^{S^{(0)}}\right]^{-1} S^{(0)}
$$

$$
S^{(1)}=\left[V I+\sigma_{0} D_{\theta \xi}^{W^{(0)}}\right]^{-1} W^{(0)}
$$

and so on.

For the given amplitude of the signal at this iteration we choose only phases of the signal according to algorithm which was described in [12]. In this work only first iteration of optimization will be considered.

If consider the case $\mathrm{M}=\mathrm{N}$ and $q_{f}=1$ in (7), (8), (9) then dimension of set of signals vector and dimension of set of filters vector are the same and equal $P N$, dimension of matrix is $P N \times P N$.

\section{Numerical Results}

We consider the case only filter optimization according to (8). As an example we calculate $W^{(0)}$ from (8) for the case $N=M=3, P=2, \sigma_{\xi}(\tau, f)=\xi \delta(f)[14]$ vector $S^{(0) t}=\left[\begin{array}{llllll}1 & -1 & -1 & 1 & 1 & 1\end{array}\right]$ and calculated filter

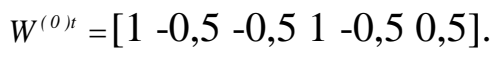


So, we have set of signals $S_{1}^{(0) t}=[1-1-1], S_{2}^{(0) t}=[111]$ and set of filters $W_{1}^{(0) t}=[1-0,5-0,5], W_{2}^{(0) t}=[1-0,50,5]$.

Cross correlation functions $R^{S_{l} W_{l}}=[-0,5 ; 0 ; 2 ;-0,5 ;-1] \quad R^{S_{2} W_{2}}=[0,5 ; 0 ; 1 ; 0,5 ; 1]$.

As we can see this pairs of signals and filters are complementary (the sum of cross correlation functions has zero side-lobes). This example is interesting because the classical Golay complementary pair for $\mathrm{N}=3$ doesn't exist, but for mismatched case it does $[19,20]$.

In this example $\mathrm{N}=\mathrm{M}=3, \mathrm{P}=2$ signal-to-noise ratio loses [19] $\rho=0,5$. But if we increase memory of filter $\mathrm{N}=3$, M=5 we get $\rho$ $=0,7$ [20]. So signal-to-noise ratio loses are decreased. For this case we have

$$
\begin{aligned}
& S_{1}^{(0) t}=[1-1-1] ; S_{2}^{(0) t}=\left[\begin{array}{lll}
1 & 1 & 1
\end{array}\right] ; \\
& W_{1}^{(0) t}=[-0,5 ; 1,0 ;-0,9 ;-0,4 ; 0,2] \\
& W_{2}^{(0) t}=[-0,5 ; 1,0 ; 0,1 ; 0,8 ;-0,2]
\end{aligned}
$$

Cross correlation functions

$R_{k}^{S_{1} W_{1}}=[0 ; 0,2 ;-0,6 ;-0,7 ; 2,3 ;-0,6 ;-0,5 ; 0,5 ; 0]$

$R_{k}^{S_{2} W_{2}}=[0 ;-0,2 ; 0,6 ; 0,7 ; 1,9 ; 0,6 ; 0,5 ;-0,5 ; 0]$.

Another example $N=5, M=5, P=2$ [20]

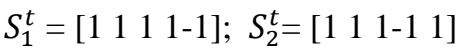

$W_{1}^{t}=\left[\begin{array}{llll}1 & 4 & 1 & 6-4\end{array}\right] ; W_{2}^{t}=\left[\begin{array}{lll}1 & 4-1-6 & 4\end{array}\right] ;$

Cross correlation functions

$\mathrm{RSW}_{1}=[-4 ; 2 ; 3 ; 7 ; 16 ; 0 ; 4 ;-3 ;-1] ;$

$\mathrm{RSW}_{2}=[4 ;-2 ;-3 ;-7 ; 14 ; 0 ;-4 ; 3 ; 1] ; \rho=0,64$.

For increased filter memory $N=5, M=7$ we have [20]

$W_{1}^{t}=[-1,5 ; 3,5 ; 2 ; 2 ; 6 ;-6,5 ;-1,5]$

$W_{2}^{t}=[-1,5 ; 3,5 ; 5 ; 1 ;-6 ; 6,5 ; 1,5] ; \rho=0,79$. So, signal-to-noise ratio loses are also decrease.

Consider a few examples else: $N=6, M=6, P=2$

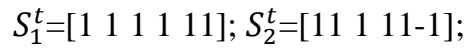

$W_{1}^{t}=[-1 ; 7 ;-1 ; 5 ; 11 ;-7] ; W_{2}^{t}=[-1 ; 7 ; 1 ;-5 ;-11 ; 7] ; \rho=0,39$.

$N=5, M=5, P=6$

$S_{1}^{t} ;=[1111-1] ; S_{2}^{t}=[111-11] \quad S_{3}^{t}=[11-111] ; S_{4}^{t}=[1-1111] ; \quad S_{5}^{t}=[-11111] ; \quad S_{6}^{t}==[11111] ; \quad \mathrm{W}_{1}=\mathrm{S}_{1} ; \quad \mathrm{W}_{2}=\mathrm{S}_{2} ; \quad \mathrm{W}_{3}=\mathrm{S}_{3} ; \quad \mathrm{W}_{4}=\mathrm{S}_{4} ;$ $\mathrm{W}_{5}=\mathrm{S}_{5} ; \mathrm{W}_{6}=-\mathrm{S}_{6} ; \rho=0,44$

$N=5, M=5, P=4$

$S_{1}^{t}=[111-11] ; S_{2}^{t}=[1-111-1] ; S_{3}^{t}=[1-1-1-11] ;$

$S_{4}^{t}=[1-1-1-1-1] ; \mathrm{W}_{1}=\mathrm{S}_{1} ; \mathrm{W}_{2}=\mathrm{S}_{2} ; \mathrm{W}_{3}=\mathrm{S}_{3} ; \mathrm{W}_{4}=\mathrm{S}_{4}$. 
$N=6, M=6, P=4$

$S_{1}^{t}=[1-111-11] ; S_{2}^{t}=[1-1111-1] ; S_{3}^{t}=[111-1-1-1] ;$

$S_{4}^{t}=[111-111]$

$\mathrm{W}_{1}=\mathrm{S}_{1} ; \mathrm{W}_{2}=\mathrm{S}_{2} ; \mathrm{W}_{3}=\mathrm{S}_{3} ; \mathrm{W}_{4}=\mathrm{S}_{4}$.

$N=7, M=7, P=8[19]$

$S_{1}^{t}=[111-1-11-1] ; S_{2}, \mathrm{~S}_{3}, \mathrm{~S}_{4}, \mathrm{~S}_{5}, \mathrm{~S}_{6}, \mathrm{~S}_{7}-$ are cyclic shifts of signal $\mathrm{S}_{1} ; S_{8}^{t}=[1111111]$;

$W_{p}=S_{p}(p=1,2, \ldots P)$.

$N=11, M=11, P=4$

$S_{1}^{t}=[111-111-11-1-11] ; S_{2}^{t}=[111-11-1-1-1-111] ;$

$S_{3}^{t}=[111-1111-111-1] ; S_{4}^{t}=[111-11-1111-1-1] ;$

$W_{p}=S_{p} \mathrm{p}(p=1,2, \ldots P) ; \quad \rho=1$.

Cross correlation functions are represented on fig.1-5.

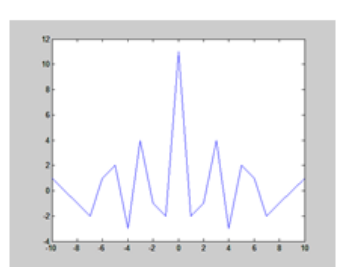

Fig.1 for $\mathrm{S}_{1}$

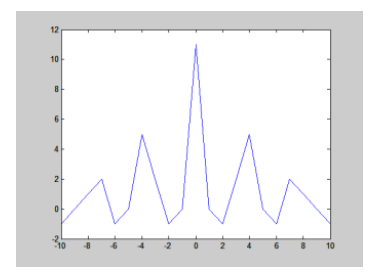

Fig.3 for $\mathrm{S}_{3}$

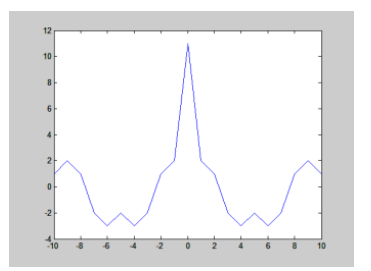

Fig.2 for $\mathrm{S}_{2}$

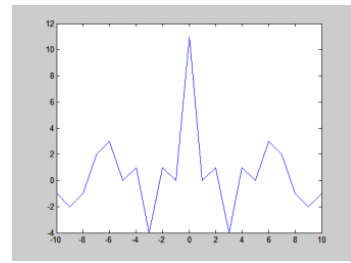

Fig.4 for $\mathrm{S}_{4}$

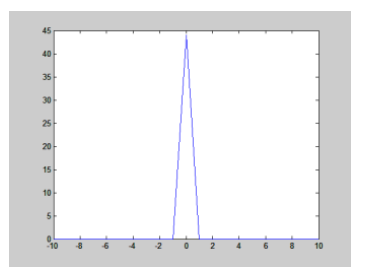

Fig. 5 for the sum of the correlation functions

In last four examples we get the maximum value of $p=1$, which corresponds to matched filters (without signal-to-noise ratio loses).

For considered cases $\sigma_{\xi}(\tau, f)=\xi \delta(f)$ matrix $D_{\theta \xi}, W^{*} S$ in (7) is formed by the next way: 


$$
D_{\theta \xi}=\xi \sum_{k=1-N}^{N-1}\left[\begin{array}{l}
S_{1 k} \\
\cdot \\
\cdot \\
S_{P k}
\end{array}\right]\left[S_{1 k}^{*} \ldots S_{P k}^{*}\right]
$$

were $S_{p k}$ - vector of signal with number p in set, which is shifted on k position:

$$
\begin{gathered}
S_{p k}=\left[\begin{array}{l}
0 \\
\cdot \\
s_{0 p} \\
s_{2 p}
\end{array}\right], \text { if } k \geq 0 ; \quad S_{p k}=\left[\begin{array}{l}
s_{N-3 p} \\
s_{N-2 p} \\
s_{N-1 p} \\
\cdot \\
0
\end{array}\right], \text { if } k \leq 0 ; \\
W * S=\left[\begin{array}{lll}
W_{1}^{*} \ldots W_{P-2}^{*} & W_{P-1}^{*} & W_{P}^{*}
\end{array}\right]\left[\begin{array}{l}
S_{1} \\
\cdot \\
S_{P-2} \\
S_{P-1} \\
S_{P}
\end{array}\right]
\end{gathered}
$$

Considered method of optimization set of filters for given set of waveforms, as a first step of joint waveform-filter sets optimization, suggested in [19], gives the possibility to get the solutions for arbitrary discrete signals and arbitrary number of signals in set. The minimum loses in signal-to-noise ratio are provided for given sets of signals. For existing non trivial solutions only no singularity of matrix (10) should be provided. For example in the case of identical signals in set the matrix (10) is singular and no trivial solutions for set of filters, which provided complementary property, doesn't exist. Increasing memory of filters leads to decreasing of signal-to-noise ratio loses. It is very important to note that getting solutions allow getting new sets of signals and filters without any additional calculations. Really, if we have set of two signals with equal $\mathrm{N}$ and set of two corresponding mismatched filters, which are complementary, we may create new complementary sets of two signals and two filters, but with twice lengths by the next way[20]: $S_{1}(2 N)=\left[S_{1}(N) ; S_{2}(N)\right] ; S_{2}(2 N)=\left[S_{1}(N) ;-S_{2}(N)\right]$;

$\mathrm{W}_{1}(2 \mathrm{~N})=\left[\mathrm{W}_{1}(\mathrm{~N}) ; \mathrm{W}_{2}(\mathrm{~N})\right] ; \mathrm{W}_{2}(2 \mathrm{~N})=\left[\mathrm{W}_{1}(\mathrm{~N}) ;-\mathrm{W}_{2}(\mathrm{~N})\right]$

The value of $p$ is reserved as for the length $\mathrm{N}$.

We can demonstrate it for example which are considered above for $N=3, M=3, P=2$

$$
\begin{aligned}
& S_{1}^{t}(6)=[1111-1-1] ; S_{2}^{t}(6)=[111-111] ; \\
& W_{1}^{t}(6)=[2-112-1-1] ; W_{2}^{t}(6)=[2-11-211] ; \rho=0,5 .
\end{aligned}
$$

If we have set of two signals with different $\mathrm{N}(\mathrm{N} 1 ; \mathrm{N} 2)$ and set of two corresponding mismatched filters, which are complementary, we may create new complementary sets of four signals and four filters with lengths $\mathrm{N}_{1}+\mathrm{N}_{2}$ by the next way[20]:

$$
\begin{aligned}
& \mathrm{S}_{1}\left(\mathrm{~N}_{1}+\mathrm{N}_{2}\right)=\left[\mathrm{S}_{1}\left(\mathrm{~N}_{1}\right) ; \mathrm{S}_{1}\left(\mathrm{~N}_{2}\right)\right] \\
& \mathrm{W}_{1}\left(\mathrm{~N}_{1}+\mathrm{N}_{2}\right)=\left[\mathrm{W}_{1}\left(\mathrm{~N}_{1}\right) ; \mathrm{W}_{1}\left(\mathrm{~N}_{2}\right)\right] ; \\
& \mathrm{S}_{2}\left(\mathrm{~N}_{1}+\mathrm{N}_{2}\right)=\left[\mathrm{S}_{1}\left(\mathrm{~N}_{1}\right) ;-\mathrm{S}_{1}\left(\mathrm{~N}_{2}\right)\right] ; \\
& \mathrm{W}_{2}\left(\mathrm{~N}_{1}+\mathrm{N}_{2}\right)=\left[\mathrm{W}_{1}\left(\mathrm{~N}_{1}\right) ;-\mathrm{W}_{1}\left(\mathrm{~N}_{2}\right)\right] ; \\
& \mathrm{S}_{3}\left(\mathrm{~N}_{1}+\mathrm{N}_{2}\right)=\left[\mathrm{S}_{2}\left(\mathrm{~N}_{1}\right) ; \mathrm{S}_{2}\left(\mathrm{~N}_{2}\right)\right] ; \\
& \mathrm{W}_{3}\left(\mathrm{~N}_{1}+\mathrm{N}_{2}\right)=\left[\mathrm{W}_{2}\left(\mathrm{~N}_{1}\right) ; \mathrm{W}_{2}\left(\mathrm{~N}_{2}\right)\right] ; \\
& \mathrm{S}_{4}\left(\mathrm{~N}_{1}+\mathrm{N}_{2}\right)=\left[\mathrm{S}_{2}\left(\mathrm{~N}_{1}\right) ;-\mathrm{S}_{2}\left(\mathrm{~N}_{2}\right)\right] ; \\
& \mathrm{W}_{4}\left(\mathrm{~N}_{1}+\mathrm{N}_{2}\right)=\left[\mathrm{W}_{2}\left(\mathrm{~N}_{1}\right) ;-\mathrm{W}_{2}\left(\mathrm{~N}_{2}\right)\right] .
\end{aligned}
$$

We can demonstrate it for examples which were calculated above for $N=M=6=N_{l}=6, P=2$ and $N=M=3=N_{2}, P=2$. 


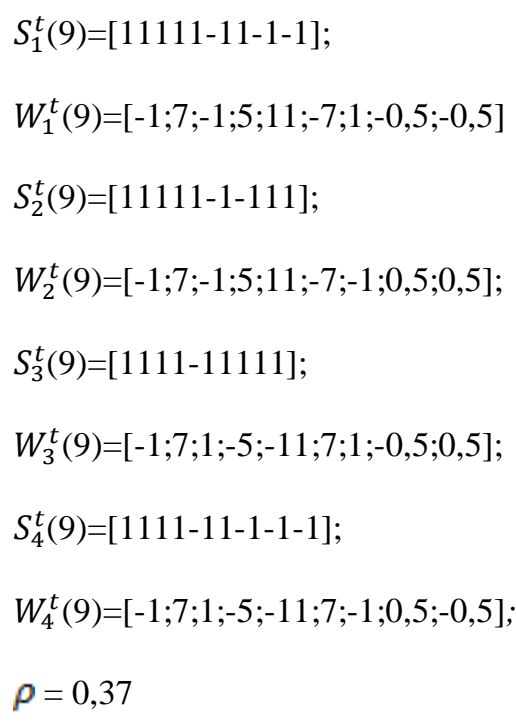

This suggested approach to construction new complementary sets of filters and signals is an extension of known for the matched filters approach for the mismatched case.

All signals and filters considered may be used for as group-complementary sets of waveforms and filters for the case of antenna with electronically scanning.

In the case of rotating antenna the group-complementary properties of sets of waveform and filters (figure a) are destroyed due to amplitude modulation. So the construction waveforms and filters may be realise in other way (figure b), which guarantees zero side-lobes level in nearby peak of correlation function zone independently of rotating antenna effect.

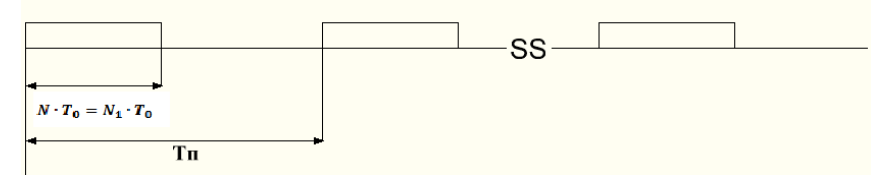

Fig.a Diagram of signals for the case of an electronic scanning antenna

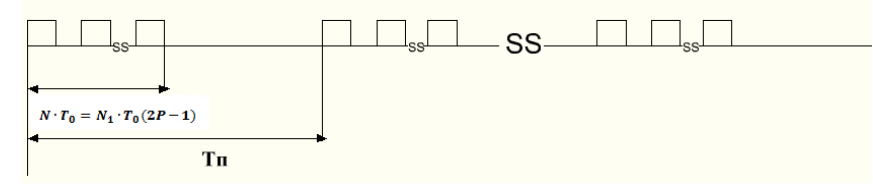

Fig.b Diagram of signals for the case of the rotating antenna

We demonstrates it on the example of $\mathrm{N}=15$ on the base of waveforms and filters set for $\mathrm{N}_{1}=5, \mathrm{M}_{1}=5$, $\mathrm{P}=2$, which we were considered aboveas the first step of approach: $S=[1111-100000111-11] ; \mathrm{W}=[1 ; 4 ; 1 ; 6 ;-4 ; 00000 ; 1 ; 4 ;-1 ;-6 ; 4]$. On the second step we choose multichannel Doppler filter according to [26]. Filter tuned on the different Doppler frequencies $l=0 \div$ $N$, $\left(l=F_{w} 4 N T_{0}\right),\left(F_{w}-\right.$ frequency of filter tuned). Cross correlation functions for these waveform and filters (for $l=0$; $l=1 ; l=2)$ are shown on Fig. 6 . On this Fig. we can see zero side-lobes level in the nearby zone of the cross correlation function central peak. $\rho=0,64$. 


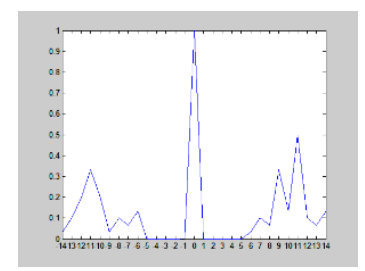

$\mathbf{l}=\mathbf{0}$

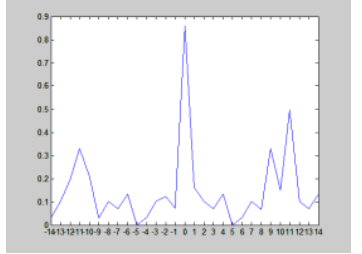

$\mathbf{l}=\mathbf{1}$

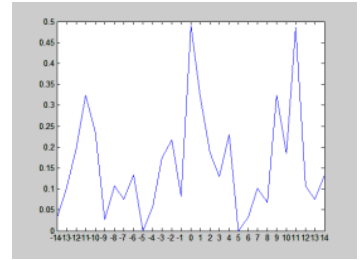

$\mathbf{l}=\mathbf{2}$

Fig. 6 When the Doppler shift of signal $11=0$ for $\mathrm{N}=15$

We also may consider the signal and filter $\mathrm{N}=77$, which are constructed on the base of waveforms and filters set for $\mathrm{N}_{1}=11 ; \mathrm{M}_{1}=11 ; \mathrm{P}=4$.

$S=[111-111-11-1-1100000000000111-11-1-1-1-11100000000000111-1111-111-100000000000111-11-1111-1-1] ;$ Cross correlation functions are shown on Fig. $7(l=0 ; l=1 ; l=2) . \rho=1$.

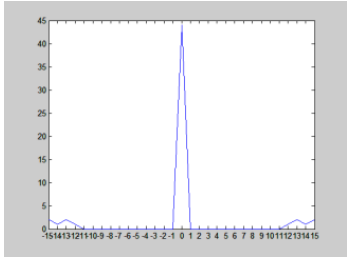

$\mathbf{l}=\mathbf{0}$

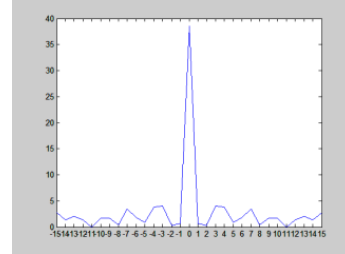

$\mathbf{l}=1$

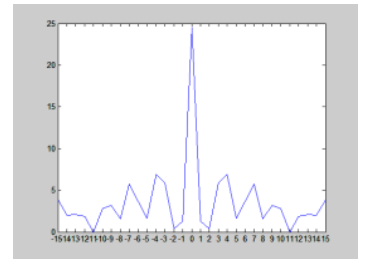

$\mathbf{l}=\mathbf{2}$

Fig. 7 When the Doppler shift of signal $11=0$ for $N=77$

The tolerance for Doppler shift of signal should be provided for both cases of antenna scanning (electronically and mechanically rotating) by means of special filter counting [26]. Results of such counting for the Doppler shift of signal $l_{l}=1$ ( $\left.l 1=F s 4 N T_{0}\right)$ are shown for last two examples on Fig.8 and Fig.9 correspondently. Tolerance to Doppler shift of signal can be seen from comparison of the cross sections $l_{l}=0$, (pictures on Fig.6, Fig.7) and $l_{l}=1$, (pictures on Fig.8, Fig.9). 


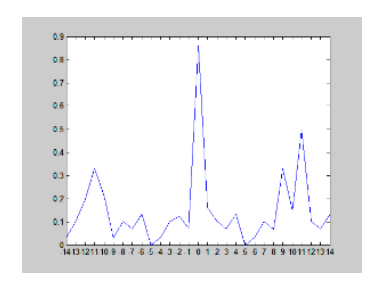

$\mathbf{l}=\mathbf{0}$

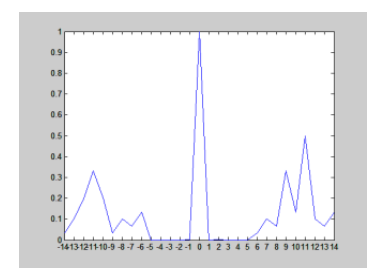

$\mathbf{l}=\mathbf{1}$

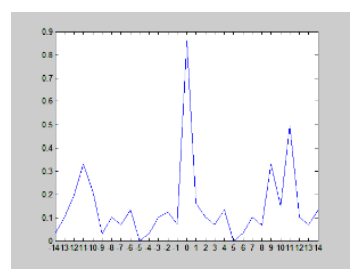

$\mathbf{l}=\mathbf{2}$

Fig. 8 When the Doppler shift of signal $11=1$ for $N=15$

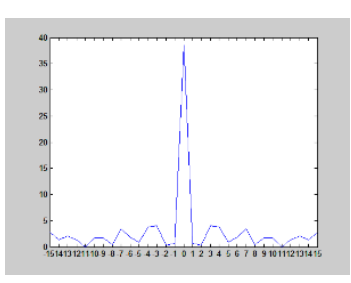

$\mathbf{l}=\mathbf{0}$

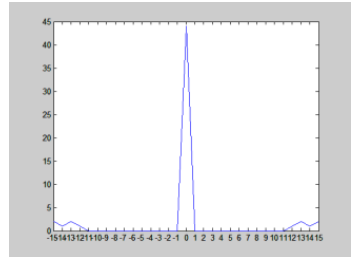

$\mathbf{l}=\mathbf{1}$

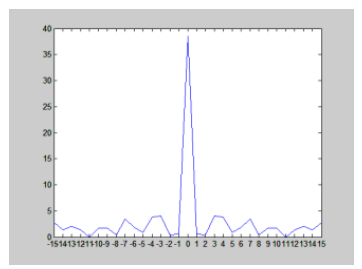

$\mathbf{l}=\mathbf{2}$

Fig. 9 When the Doppler shift of signal $11=1$ for $N=77$

Third step of approach, connected with decreasing range side-lobes in the Doppler strip nearby zero Doppler frequency, can be demonstrated on the base of example for $\mathrm{N}=5, \mathrm{M}=5, \mathrm{P}=2$, considered above. For this purpose we choose four blocks of such waveforms and filters $S_{1}, S_{2}, W_{1}, W_{2}$, with next dislocation: $S^{t}=\left[S_{1}^{t} 00000 S_{2}^{t} 00000 S_{2}^{t} 00000 S_{1}^{t}\right]$, and $W^{t}=$ $\left[W_{1}^{t} 00000 W_{2}^{t} 00000 W_{2}^{t} 00000 W_{1}^{t}\right]$.

Explanation of such kind of dislocation may be given by the next. Really in each block mutual subtraction of range sidelobes of aperiodic cross correlation functions and summation of their main lobes are provided due to their complementary property. So relatively to the range side lobes between periods subtractions are created of the one group of aperiodic cross correlation functions side lobes summation (which represents one group of waveforms and corresponding filters) from another group aperiodic cross correlation side lobes summation (corresponded to another group of waveforms and filters). Thus we may denotes one group of waveforms and corresponding filters as 1 and another group of waveforms and corresponding filters as -1. Such approach is used for the case of two waveforms in block under matched filtering (Golay complementary pairs) with dislocation blocks according to PTM sequences in [18].

Appointed above subtraction may be interpreted as passing the summation of aperiodic cross correlation functions side-lobes of one group waveforms and corresponding filters and the summation of aperiodic cross correlation functions side-lobs for another group waveforms and corresponding filters through Single moving-target indicator (MTI) (with delay T) Canceler with weights 1 and -1 correspondingly. 
So in one block of waveforms and filters is provided simple subtraction of side-lobs and that is equivalent of their passing through single canceler [2,3] and corresponding initial dislocation two groups of waveforms and filters in block is

$$
--11
$$

For providing double subtraction two blocs of waveforms and corresponding filters are needed. From the difference of first block subtraction the difference of second block should be provided. This is equivalent to the passing through double canceler. Corresponding dislocation groups in two blocks is

$$
--111--1
$$

For realization third order subtraction and corresponding third order canceler four blocks are needed with next dislocation groups in them

$$
--1111--11--1--11
$$

For the forth order we have

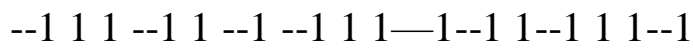

And so on ...

Number of blocks is needed for m order canceler is

$$
P_{1}=2^{m-1}
$$

Redoubling number of blocks at each following subtraction is needed for increasing of canceler order. It follows from (16). Dislocation of groups in blocks also has very simple structure. Under each increasing of canceler order new dislocation repeats previous one with adding the same dislocation with opposite sign.

The magnitude of the frequency response which corresponded $m$ order subtraction is given by

$$
|H(f)|^{m}=\left|\left(1-e^{-j 2 \pi f T}\right)\right|^{m}=2^{m}|\sin (\pi f T)|^{m}
$$

Expression (17) gives only limited characteristics of $\mathrm{m}$ order subtraction and depends from concrete composition of waveforms in the block and order of disposition of signals of in each block. As can be seen from (17) with increasing order of subtraction the magnitude of frequency response nearby Doppler frequency $f=0$ is flatting, and correspondingly the width of Doppler side lobes suppression strip is increased. It is confirmed by considered example of waveforms disposition corresponded (13) (second order canceler) and results of calculations which are presented on Fig.10 and Fig.11.

Comparison Fig.10 and Fig.11 with Fig.6 and Fig.8 (which corresponded first order canceler) shows significant decreasing of side lobes level in Doppler strip we have got by means increasing number of blocks with proper choosing order of their dislocation in each block. 


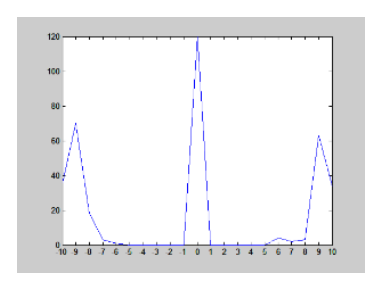

$\mathbf{l}=\mathbf{0}$

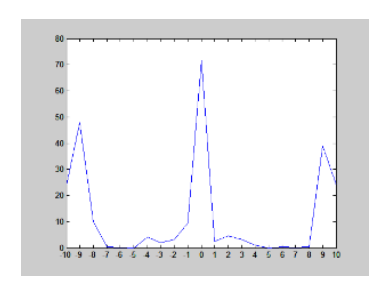

$\mathbf{l}=\mathbf{2}$

Fig.10 When the Doppler shift of signal $11=0$ for $\mathrm{N}=75$

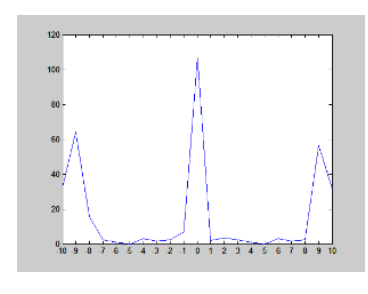

$\mathbf{l}=\mathbf{0}$

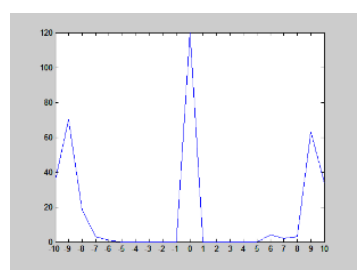

$\mathbf{l}=\mathbf{1}$

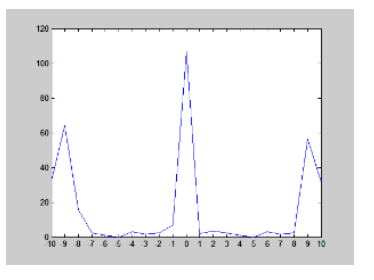

$\mathbf{l}=\mathbf{2}$

Fig.11 When the Doppler shift of signal $l_{1}=1$ for $N=75$

The results of calculation for example on the base of $\mathrm{N}=3, \mathrm{M}=3, \mathrm{P}=2$ with dislocation of waveforms and filters according to (13) also shows good coordination with expression (17).

\section{References}

[1] Marine radar. Edited by V.I. Vinokurov. (In Russian) - L. Sudoctroenie, 1986.

[2] Radar Handbook. Editor-In-Chief M.I. Skolnik. McGraw-Hill Book Company, 1970.

[3] N. Levanon, E. Mozeson. Radar Signals. - Wiley-Interscience, 2004.

[4] W.D.Rummler. 'Clutter Suppression by Complex Weighting of Coherent Pulse Trains.'IEEE Trans. on AES. Vol. AES2. No.6. pp. 810-818. Nov.1966.

[5] W.D.Rummler. "A Technique for Improving the Clutter Performance of Coherent Pulse Train Signals.”IEEE Trans. on AES. vol. AES-3. No.6. pp. 898-906. Nov. 1967.

[6] D.F. Delong. Jr. and E.M. Hofstetter. ' On the Design of Optimum Radar Waveforms for Clutter rejection.” IEEE Trans. on IT. Vol. IT-13. No.3. pp. 454-463. July 1967. 

1968.

[7] L.J. Spafford. "Optimum Radar Signal Processing in Clutter.” IEEE Trans. on IT. Vol. I14. No.5. pp. 734-743. Sept.

[8] C.A. Statt and L.J. Spafford. "A "Best" Mismatched Filter Response for Radar Clutter Discrimination ." IEEE Trans. on IT. vol. IT-14. No. 2. pp. 280-287. Mar. 1968.

[9] V.T. Dolgochub and M.B. Sverdlik. “Generalized v-filters.” Radio Engeneering and Electronic Physics. Vol. 15. pp.147150. January 1970.

[10] Y.I. Abramovich and Sverdlik. "Synthesis of a filter which maximizes the signsl-to-noise ratio under additional quadratic constraints.’ Radio Engineering and Electronic Physics. vol. 15. pp. 1977-1984. Nov. 1970.

[11] P. Stoica, J. Li, M. Xue. "Transmit Codes and Receive Filters for Pulse Compression Radar Systems.” IEEE Signal Processing Mag. vol. 25. no. 6. pp.815-845. Nov. 2008.

[12] V.M. Koshevyy, M.B Sverdlik .'Synthesis of Signal - Filer pair under additional constraints'. Radio Engineering and Electronics. vol 21. no.6. pp. 1227-1234. June 1976.

[13] M.J.E. Golay,”Complementary series,” IRE Trans. Inform. Theory, vol. IT-7, pp 82-87, Apr. 1961.

[14] C.C. Tsehg, C.L. Liu, “Complementary sets of sequences,” IEEE Trans. Inform. Theory, vol. IT-18, no. 5, pp. 644-652, Sept. 1972.

[15] S.D. Howard, A.R. Calderbank, W. Moran,"A Simple Ploarization Diversity Scheme for Radar Detection," Proc. of Second Int. Conf. on waveform Diversity and Design, Lihue, HI, pp. 22-27, Jan. 2006.

[16] S. Alamouti,"A Simple Transmit Diversity Technique for Wirless Communication,” IEEE J. Select. Areas Comm., vol. 16, , no. 8, pp.1451-1458, Oct. 1998.

[17] T.R. Qureshi, M.D. Zolowski, R. Caldebank,'Target detection in MIMO radarin the presence of Doppler using complementary sequences," Proc. ICASSP-2010,pp.2766-2769.

[18] A. Pezeshki, A. R. Calderbank, W. Moran, S. D. Howard,'Doppler resilient Golay complementary waveforms," IEEE Trans. Inform. Theory, vol. 54, no. 9, pp.4254-4266, Sep. 2008.

[19] V.M. Koshevyy. "Synthesis of Waveform-Filter pairs under Additional Constraints with Group-Complementary Properties” IEEE, Radar Conference 2015, May 2015, Arlington,VA (USA), pp.0616-0621.

[20] V.M. Koshevyy. "Efficiency of Filter Synthesis under Additional Constaints with Group-Complementary Properties" IEEE, 2016 International Conference "Radioelectronics \& InfoCommunications" (UkrMiCo), September 11-16, 2016, Kiev, Ukraine, pp. 978-982/

[21] A.W. Rihachek. Principles of High Resolution Radar. New York: Mc Graw-Hill. 1969 .pp. 153.

[22] V.M. Koshevyy. Synthesis of multi frequency codes under additional constraints Radio Engineering and Electronics. Vol 29.N11 . 1984.

[23] V.M. Koshevyy, M.B. Sverdlik. Joint ptimization of Signal and Filter in the Problems of Extraction of Signals from Interfering Reflections. Radio Engineering and Electronic Physics. Vol.20. N 10 pp.48-55.

[24] Glenn Weathers, Edvard M. Holiday. Group-Complementary Array Coding for Radar Clutter Rejection. IEEE Trans. On Aerospace and Electronic System . Vol . AES-19. N3 May 1983.

[25] Bi, J. Rohling, H. Complementary Binary Code Design based on Mismatched Filter. IEEE Trans. on Aerospace and Electronic System, Vol. 48, N2, January 2012.

[26] V.M. Koshevyy, M.B. Sverdlik,“ On one property of an optimum signal” Radio Engineering and Electronic Physics. 1977, No. 10, Vol. 22, pp.73-75.

[27] V. Koshevyy, V. Popova "Complementary coded waveforms sets in marine radar application". IEEE first Ukraine conference on electrical and computer engineering ( UKRCON), 2017, pp.215 - 220. 